\title{
Topographic anatomy of the eyelids, and the effects of sex and age
}

Department of Oculoplastic Surgery, The Rotterdam Eye Hospital, Rotterdam, Netherlands

W A van den Bosch

Havenziekenhuis, Rotterdam, Netherlands I Leenders

Department of Epidemiology and Biostatistics, Erasmus University Medical School, Rotterdam, Netherlands

P Mulder

Correspondence to: Willem A van den Bosch $\mathrm{MD}$, The Rotterdam Eye Hospital, PO Box 70030 7000 LM Rotterdam, Netherlands.

Accepted for publication 15 October 1998

Willem A van den Bosch, Ineke Leenders, Paul Mulder

\begin{abstract}
Aims-To describe the effects of sex and age on eyeball, eyelid, and eyebrow position.

Methods-A cross sectional cohort study was performed in which both eyes of 320 normal subjects aged between 10 and 89 years were included. Of each 10 year age cohort, there were 20 men and 20 women. Frontal, as well as lateral, slides were taken of both eyes. On projected slides, a reference line through the medial canthi and vertical lines through the pupil centre and the lateral canthus were constructed. Using these lines, we measured the size of the horizontal eyelid fissure, the distance from the reference line to the pupil centre and to the lateral canthus, the distance between the pupil centre and the upper and lower eyelid margin, and the distance between the upper eyelid margin and the skin fold and eyebrow. On lateral slides, the distance between the lateral canthus and the anterior corneal surface was measured.
\end{abstract}

Results-Between the ages of approximately 12 and 25 years, the horizontal eyelid fissure lengthened $3 \mathrm{~mm}$, while the position of other eyelid structures remained virtually unchanged. Between the average ages of 35 and 85 years, the horizontal eyelid fissure gradually shortened again by about $2.5 \mathrm{~mm}$. Meanwhile, the distance between the lateral canthal angle and the anterior corneal surface decreased almost $1.5 \mathrm{~mm}$. Aging caused an increase of the distance between the pupil centre and the lower eyelid of about $1 \mathrm{~mm}$ in men, and $0.5 \mathrm{~mm}$ in women. Aging also caused a higher skin crease and raised eyebrows in men and women, but it did not affect the position of the pupil centre and the lateral canthus. Men showed an $0.7 \mathrm{~mm}$ larger horizontal eyelid fissure than women. In women, however, the eyebrows were situated about $2.5 \mathrm{~mm}$ higher than in men.

Conclusion-Aging mainly affects the size of the horizontal eyelid fissure, which lengthens by about $10 \%$ between the ages of 12 and 25, and shortens by almost the same amount between middle age and old age. Aging causes sagging of the lower eyelid, especially in men, and a higher skin fold and eyebrow position in both sexes. Aging does not affect the position of the eyeball proper, or of the lateral canthus. (Br F Ophthalmol 1999;83:347-352)
Aging may cause laxity of eyelid tissues and atrophy of the orbital fat. These changes contribute to the aetiology of several eyelid disorders such as ectropion, entropion, dermatochalasis, and aponeurogenic blepharoptosis. ${ }^{1-4}$

Such aging changes may also affect the position of the eyelids, eyeball, and eyebrow. Knowledge of normal eyelid position in different age groups might therefore be helpful in the understanding of age related eyelid pathology.

Current studies on topographic eyelid anatomy present few data on the effects of aging. Also, these studies have not related the position of the eyelids to the position of the eyeball. ${ }^{5-13}$ Some other studies described the position of the eyeball relative to adjacent osseous structures, but did not relate the position of the eyeball to the position of the eyelids. ${ }^{14-17}$

Although the eyelids are three dimensional structures, their topographic anatomy has, to our knowledge, only been studied in a frontal plane. We speculate that several diseases, such as age related orbital fat atrophy and Graves' orbitopathy, might also affect the relation between eyelids and eyeball in an axial plane.

Some authors have postulated that aging causes a downward displacement of the lateral canthus and of the eyeball proper. This has, however, not been substantiated by any measurements. ${ }^{18-20}$

In the current study, we provide data on eyeball and eyelid position of 160 men and as many women, equally divided in 10 year age cohorts between the ages of 10 and 89 years.

\section{Subjects and methods}

The second author (IL) recruited normal white subjects from the staff and outpatients of a general hospital, except for the youngest group of subjects, who were largely recruited from two classes of a primary school. The age of the subjects ranged between 10 and 89 years of age. They were included after informed consent had been obtained. Family members of participating subjects were excluded. Subjects were also excluded who suffered, or had suffered, from any disease that might affect the eye or eyelid position, such as Graves' orbitopathy, neurological disorders, generalised muscular disease, and the wearing of hard contact lenses. We also excluded subjects who had undergone any ocular or eyelid surgery. In each age group with a bin width of 10 years, we included 20 men and 20 women. In total, 320 subjects were included.

The average age of the youngest subjects was 12 years. In all other cohorts, the ages were more evenly spread. It proved difficult to include subjects aged between 80 and 89 years, 

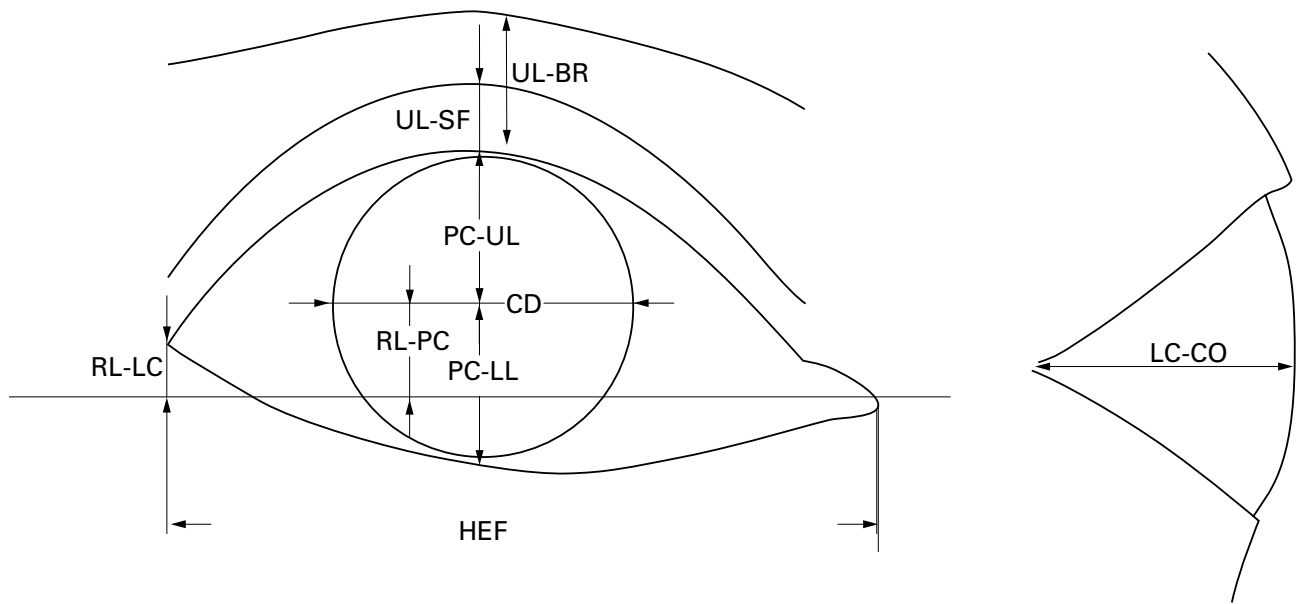

Figure 1 Schematic representation of the measurements. Left: frontal slides. A reference line was drawn through the medial canthus on both sides, vertical lines were drawn through the pupil centre and through the lateral canthal angle. HEF = horizontal eyelid fissure, $R L-P C=$ reference line to pupil centre, $P C-U L=$ pupil centre to upper eyelid margin, $P C-L L=p u p i l$

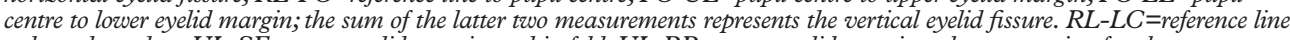
to lateral canthus, $U L-S F=$ upper eyelid margin to skin fold, $U L-B R=u p p e r$ eyelid margin to lower margin of eyebrow, $C D=$ corneal diameter. Right: lateral slides. $L C-C O=$ distance between lateral canthus and anterior corneal surface.

because cataract surgery had been performed in a large majority of the potential subjects.

For each subject, slides were made under normal interior light conditions, with a camera mounted with a $135 \mathrm{~mm}$ macro lens and a flash unit. Slides in a frontal plane were made while the subjects looked into the camera lens with their head in normal position. They held a ruler horizontally on the forehead. Great care was taken to align the camera with the eyes of the subject in a horizontal plane. Both eyes were pictured on the same slide. Slides from the left and right side in a lateral plane were made while the subjects looked straight ahead and held the ruler by the medial canthus. To obtain an angle of about 90 degrees with the anterior plane, these slides were made when the subjects were seen in profile.

Measurements were taken on these slides, being projected with threefold magnification. The ruler was used for calibration. On frontal slides, a reference line through the most nasal point of both medial canthal angles was drawn and a vertical line was drawn through the pupil centre. With this line, the distance between the reference line and the pupil centre (RL-PC) was measured. With the pupil centre as a refer-
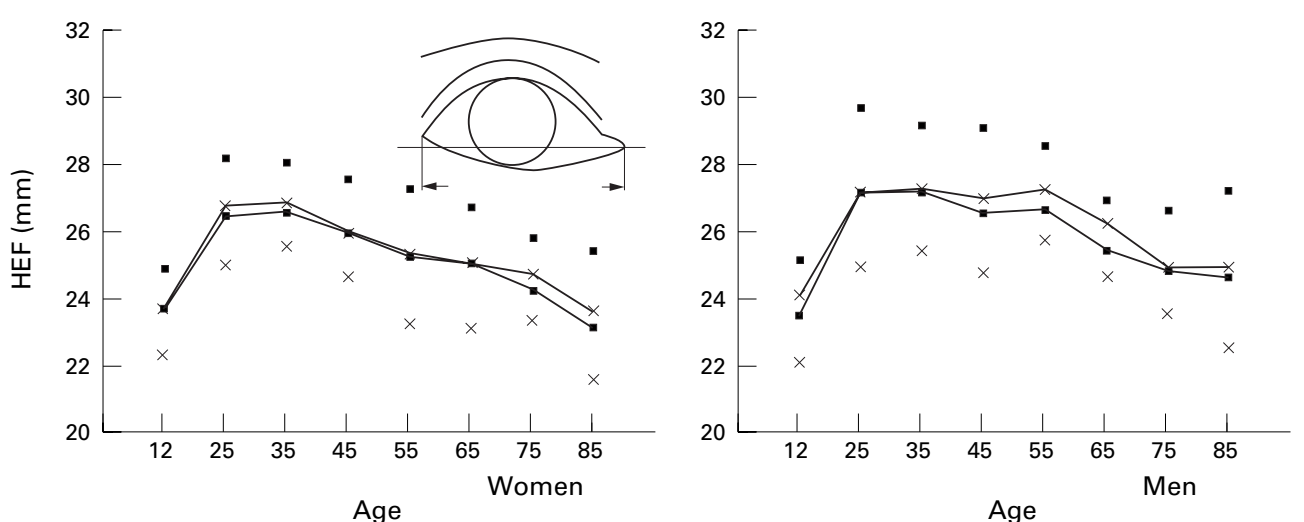

Figure 2 Horizontal eyelid fissure size (HEF) in women (left) and men (right). Horizontal axis: average age of each 10 year cohort. Vertical axis: size of the horizontal eyelid fissure ( $\mathrm{mm}$ ). Squares: right eyes, crosses: left eyes. The lines represent the average values, the symbols above and below the lines represent the average value $+1 S D$ for right eyes and the average value -1 SD for left eyes, respectively.

ence, the distance to the upper (PC-UL) and lower eyelid margin (PC-LL) was measured. The vertical line through the pupil centre was also used to measure the distance between the upper eyelid margin and the lower border of the upper eyelid skin fold (UL-SF), defined as the lowermost border of the double layer of skin that overhangs the pretarsal space in primary position of gaze, and between the upper eyelid margin and the lower margin of the eyebrow (UL-BR), defined as the lowest point where the eyebrow lashes insert. ${ }^{9}$ A second vertical line was drawn through the lateral canthal angle. This line was used to measure the distance between the reference line and the lateral canthus (RL-LC). The size of the horizontal eyelid fissure (HEF) was expressed as the distance between the most nasal point of the medial canthus and the intersection of the vertical line through the lateral canthus with the reference line. The horizontal corneal diameter was measured (CD). On lateral slides, we measured the distance between the lateral canthal angle and the anterior corneal surface (LC-CO). Figure 1 shows the measurements schematically. 

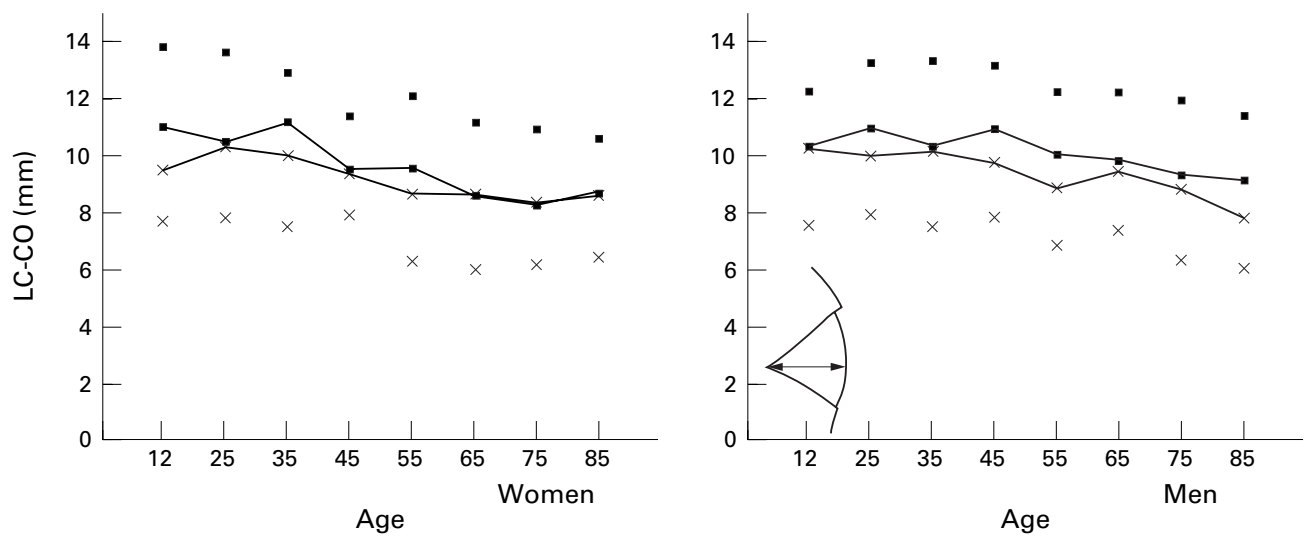

Figure 3 Distance measured from the side between the lateral canthal angle and the anterior corneal surface (LC-CO) in women (left) and men (right). For legend see Figure 2.

All measurements were taken by the same technician who was unaware of any working hypothesis of the study. The precision of the measurements was calculated by repeating them on the slides of 10 randomly selected subjects. The within subject standard deviation estimated from both eyes of these subjects proved a constant factor; it ranged from 0.24 to $0.32 \mathrm{~mm}$ across the nine variables considered.

Statistical analysis was performed by means of the rm (repeated measures) ANOVA. The repeated measures were those from the right and left side of each subject. A linear relation between the dependent variables and the independent variables age, sex, and their interaction was estimated and tested. For the horizontal eyelid fissure a polynomial (up to the fourth degree) relation with age was used, because of its concave form.

\section{Results}

The HEF lengthened by more than $10 \%$ between the mean ages of 12 and 25 years in both sexes (Fig 2). In women, its mean length was $23.7 \mathrm{~mm}$ (SD $1.3 \mathrm{~mm}$ ) at the age of 12 years, and $26.7 \mathrm{~mm}(1.8 \mathrm{~mm})$ at the mean age of 25 years. In men, the increase in length was larger; at the mean age of 12 years it measured about $23.8 \mathrm{~mm}(1.9 \mathrm{~mm})$, while it averaged $27.2 \mathrm{~mm}(2.4 \mathrm{~mm})$ at the mean age of 25 years. We found no significant change in the position of other eyelid structures between the average ages of 12 and 25 years.

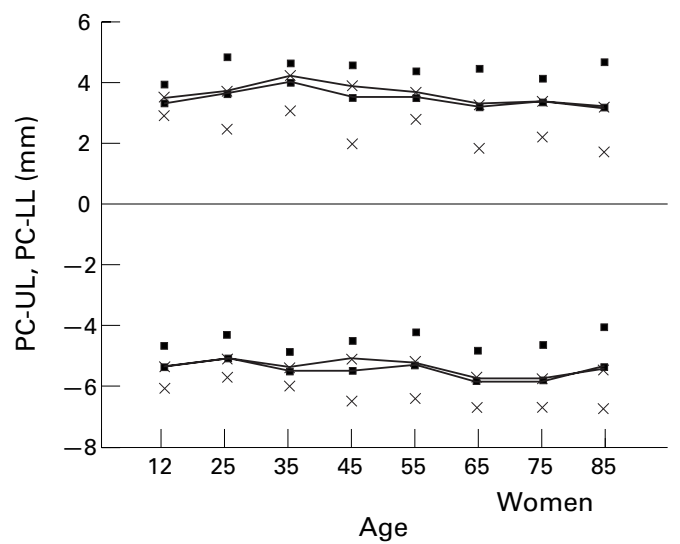

From the mean age of 45 years, the horizontal eyelid shortened again, eventually becoming, at the mean age of $85,23.5 \mathrm{~mm}$ (SD 2.1 $\mathrm{mm}$ ) in women and $24.8 \mathrm{~mm}$ (SD $2.5 \mathrm{~mm}$ ) in men. All linear and higher order effects for describing the concave relation with age were different from zero $(p=0.01)$. The initial lengthening and the shortening thereafter were similar for the two sexes (Fig 2). There was only a main effect of sex: the size of the horizontal eyelid fissure was slightly larger in men than in women $(p=0.01)$. In men, it averaged $25.8 \mathrm{~mm}$ (SD $2.4 \mathrm{~mm}$ ), and in women $25.1 \mathrm{~mm}$ (SD $2.1 \mathrm{~mm}$ ).

The distance between the lateral canthus and the anterior corneal surface (LC-CO) showed a gradual decrease with age (Fig 3). Between the ages of 25 and 85 years it decreased by almost $1.5 \mathrm{~mm}$ in men and women alike $(\mathrm{p}=0.0001)$.

The distance between the pupil centre and the upper eyelid margin (PC-UL) showed a small but not significant downward shift with age in both men and women. However, in both sexes, the distance between pupil centre and lower eyelid margin (PC-LL) increased between the ages of 25 and 85 years $(p=0.0003)$. This increase was larger in men than in women $(p=0.02)$; in men the distance between pupil centre and lower eyelid margin increased almost $1.0 \mathrm{~mm}$ between the ages of 25 and 85 years compared with about $0.5 \mathrm{~mm}$ in women (Fig 4).

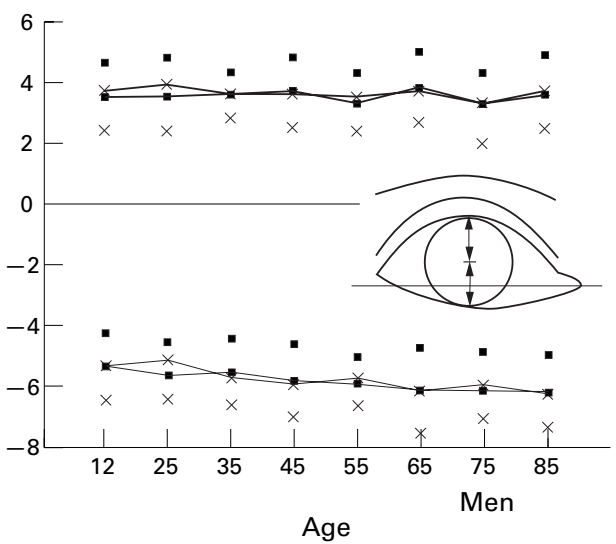

Figure 4 Distance between the pupil centre and the upper eyelid margin (PC-UL; positive values) and between the pupil centre and the lower eyelid margin (PC-LL; negative values) in women (left) and men (right). For legend see Figure 2. 

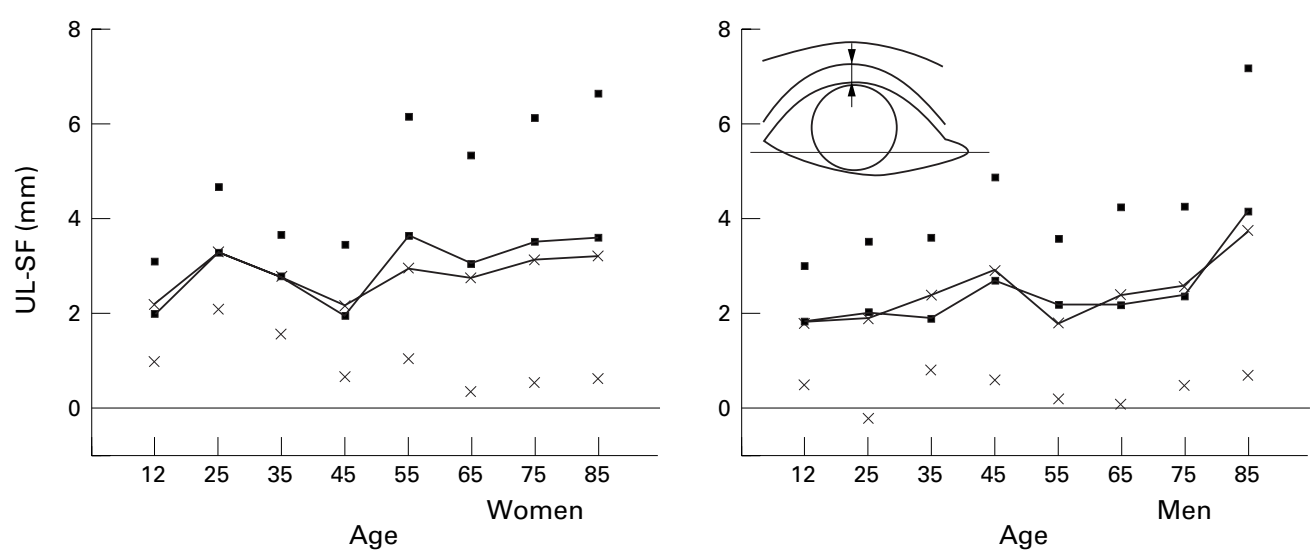

Figure 5 Distance between the upper eyelid margin and the skin fold (UL-SF) in women (left) and men (right). For legend see Figure 2.

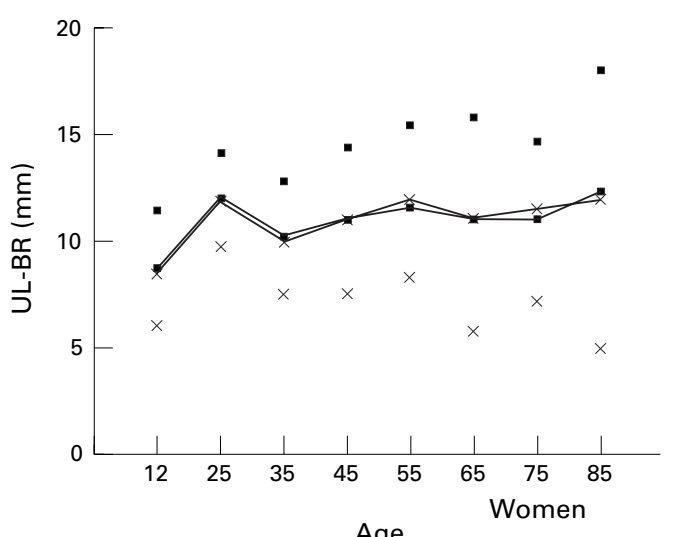

Age

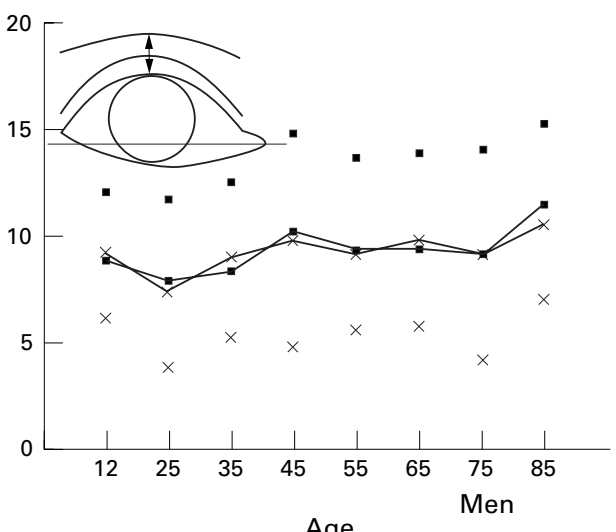

Age

Figure 6 Distance between the upper eyelid margin and the lower margin of the eyebrow (UL-BR) in women (left) and men (right). For legend see Figure 2.
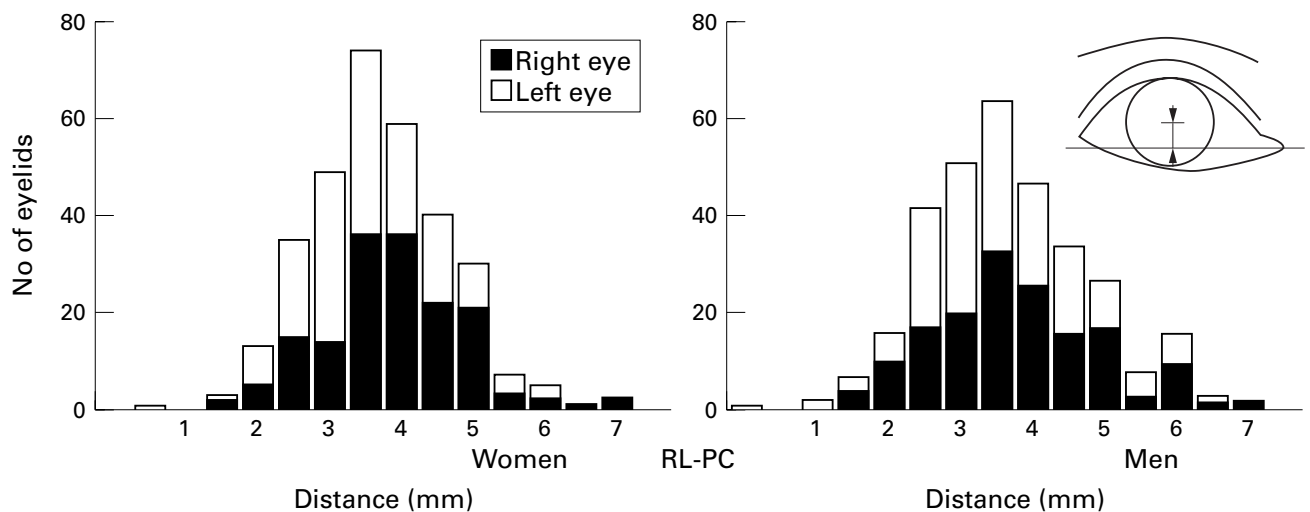

Distance $(\mathrm{mm})$

Figure 7 Distribution of the distance between the reference line and the pupil centre (RL-PC) in women (left) and men (right). Horizontal axis: distance in $\mathrm{mm}$. Vertical axis: number of eyelids.

In both sexes, the distance between the upper eyelid margin and the skin fold (UL-SF) increased with age by the same trend $(p=0.0004)$. In men, especially, the oldest age group showed a sudden increase of their skin fold height. On average, women showed a larger distance between the upper eyelid margin and the skin fold than men did; it averaged $3.0 \mathrm{~mm}$ (SD $2.0 \mathrm{~mm}$ ) in women, and 2.1 $\mathrm{mm}(2.2 \mathrm{~mm})$ in men $(\mathrm{p}=0.02)$ (Fig 5).

The distance between the upper eyelid margin and the eyebrow (UL-BR) increased with age in both men and women by the same trend $(p=0.002)$. In women, the position of the eyebrow was higher than in men; their average distance between the upper eyelid margin and the eyebrow was $11.8 \mathrm{~mm}(3.9 \mathrm{~mm})$, in men it averaged $9.4 \mathrm{~mm}(4.1 \mathrm{~mm})(\mathrm{p}=0.0001)$ (Fig 6).

The distance between the reference line and the pupil centre (RL-PC) was equal in both sexes and showed no change with age; it averaged $3.7 \mathrm{~mm}(1.1 \mathrm{~mm})$ across all ages. Figure 7 shows its distribution for the two sexes.

The distance between the reference line and the lateral canthal angle (RL-LC) was also not affected by age; it also showed no difference between the sexes. It averaged $1.9 \mathrm{~mm}(1.4$ $\mathrm{mm})$ in women, and $2.0 \mathrm{~mm}(1.5 \mathrm{~mm})$ in men over all ages (Fig 8). 

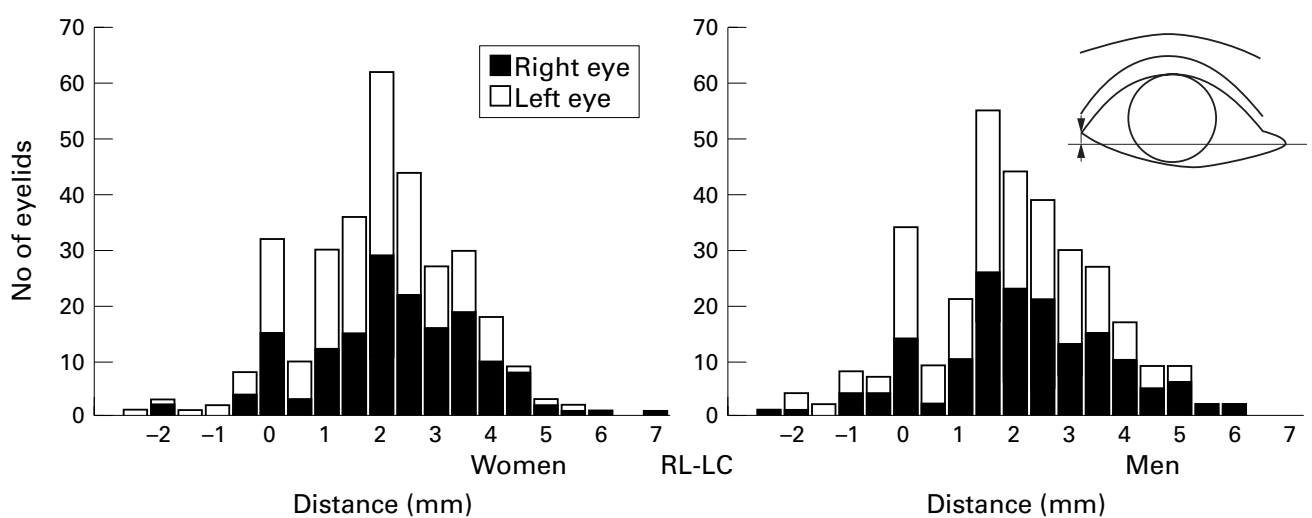

Figure 8 Distribution of the distance between the reference line and the lateral canthus (RL-LC) in women (left) and men (right). For legend see Figure 7.

The horizontal corneal diameter (CD) showed no significant difference between men and women, nor did age affect its size; it averaged $11.8 \mathrm{~mm}(0.6 \mathrm{~mm})$ in women and 11.9 $\mathrm{mm}(0.7 \mathrm{~mm})$ in men across all ages.

\section{Discussion}

Aging primarily affects the size of the horizontal eyelid fissure. We found that it lengthens by more than $10 \%$ between approximately 12 and 25 years of age. From approximately 45 years old it gradually shortens by the same amount. These data confirm those of Hill, who found that subjects above 50 years of age have a smaller size of the horizontal eyelid fissure than at younger age. ${ }^{67}$ By contrast, Fox stated that the growth of the horizontal eyelid fissure is complete at the age of 10 years, and that some shortening may occur after the age of $60 .^{5} \mathrm{Liu}$ and Stasior reported no difference in the size of the lower eyelid between subjects younger and older than 50 years of age. However, they did not measure the entire length of the lower eyelid, but the distance between the lateral canthal angle and the lower lacrimal punctum. Furthermore, they judged the distance from a ruler held near the lower eyelid margin, which presumably may have been less precise than our measurements. ${ }^{8}$

While the lengthening of the horizontal eyelid fissure between the ages of 12 and 25 years probably reflects growth of the facial structures, the shortening from the age of 45 years onwards is likely to be due to progressive laxity of the medial and lateral canthal structures. The occurrence of lateral canthal laxity may also explain the gradual decrease of the distance between the lateral canthal angle and the anterior corneal surface, as measured from the side: because the eyeball is round, medial displacement of the lateral canthus, in frontal view, would at the same time yield its forward displacement, as viewed from the side.

A similar decrease of the distance between the lateral canthal angle and the anterior corneal surface, as viewed from the side, might also be caused by involutional enophthalmos, due to atrophy of the orbital fat. ${ }^{21}$ Since our slides did not enable us to measure the distance between the anterior corneal surface and the lateral orbital wall, we cannot differentiate between these two mechanisms. However, a study in which the latter distance was measured failed to demonstrate any age related enophthalmos. ${ }^{7}$ Its role, therefore, remains unclear. Regardless which of the two mechanisms described above plays a role, the age related shortening of the horizontal eyelid fissure, as measured from anterior, and the decrease of the distance between the lateral canthus and the anterior corneal surface, as measured from the side, probably reflect progressively laxity of the eyelids with age. ${ }^{7822}$

We also found that the distance between the reference line and the lateral canthus, and between the reference line and the pupil centre, respectively, remain stable throughout life, and are identical for the sexes. This contrasts with previous assumptions that the lateral canthus is situated higher in women, and that aging causes a downward displacement of both the eyeball and the lateral canthus. ${ }^{18-20}$ Our findings do not preclude any such changes in some individuals. However, we question if the lateral canthus should routinely be put higher during lower eyelid blepharoplasty. ${ }^{18}$

Aging causes a downward shift of the lower eyelid. Interestingly, this downward shift is larger in men than in women. This suggests that aging causes more lower eyelid laxity in men. It would therefore be of interest to investigate if men suffer more frequently from lower eyelid disorders which are related to excessive laxity, such as involutional entropion or ectropion.

Aging also causes a higher skin crease in both sexes. This may be due to age related disinsertion of the levator muscle aponeurosis, and to involutional atrophy of the orbital fat. It may also be that it occurs secondary to the upward shift of the eyebrows we found in both men and women.

This upward shift of the eyebrow with aging that we found contradicts the generally held assumption that aging causes a lower position of the eyebrow. We speculate that age related laxity of the forehead skin may be compensated for by increased frontalis muscle action. This might cause wrinkling of the forehead skin, while it prevents lowering of the eyebrows. We cannot rule out that, in women, brow alopecia or epilation of the lowermost eyebrow lashes may have influenced our measurements. We do 
not, however, assume that this played any role in men, who showed an upward shift of the eyebrow by the same trend as women did.

Since our study design was cross sectional, our data may obviously not reflect the effect of aging in individual subjects.

In summary, we have demonstrated that the horizontal eyelid fissure grows by more than $10 \%$ of its length between puberty and adolescence, and that its size diminishes almost as much between middle age and old age. This decrease in its size is related to a decrease in the distance from the side between lateral canthus and anterior corneal surface, which means that the shortening of the horizontal eyelid fissure can at least partially be attributed to medial displacement of the lateral canthus. The positions of the lateral canthus and of the pupil centre are identical in men and women, and remain fairly stable throughout life. Aging causes sagging of the lower eyelid, especially in men, and a higher position of the skin crease and eyebrow in both sexes.

We hope that our data may prove helpful in the evaluation of disorders of eye and eyelid position. However, the normal values in this study may only be representative for European white subjects. Furthermore, we do not know which part of their pseudo-Gaussian distribution represents a cosmetically desirable eyelid position. Since oculoplastic surgeons often strive to accomplish this, it might be of interest to conduct a comparable study on beautiful eyelids.

The authors are grateful to Willem van den Bosch $\mathrm{Sr}$ for performing the measurements.

1 Jones LT, Reeh MJ, Tsujimura JK. Senile entropion. Am $\mathcal{F}$ Ophthalmol 1963;55:463-9.
2 Dryden RM, Leibsohn J, Wobig J. Senile entropion. Pathogenesis and treatment. Arch Ophthalmol 1978;96:1883-5.

3 Benger RS, Frueh BR. Involutional ectropion: a review of the management. Ophthalmic Surg 1987;18:136-9.

4 Dortzbach RK, Sutula FC. Involutional blepharoptosis. A histopathologic study. Arch Ophthalmol 1980;98:2045-9.

5 Fox SA. The palpebral fissure. Am 7 Ophthalmol 1966;62: $73-8$.

6 Hill JC. An analysis of senile changes in the palpebral fissure. Can f Ophthalmol 1975;10:32-5.

7 Hill JC. Analysis of senile changes in the palpebral fissure. Trans Ophthalmol Soc UK 1975;95:49-53.

8 Liu D, Stasior OG. Lower eyelid laxity and ocular symptoms. Am f Ophthalmol 1983;95:545-51.

9 Cartwright MJ, Kurumety UR, Nelson CC, et al. Measurements of upper eyelid and eyebrow dimensions in healthy white individuals. Am f Ophthalmol 1994;117:231-4.

10 Zamora RL, Becker WL, Custer PC. Normal eyelid crease position in children. Ophthalmic Surgery 1994;25:42-7.

11 Meyer BC. Incidence of a difference in size of palpebral fissure in 500 normal cases. Arch Neurol Psychiatry 1947;57: $464-6$.

12 Fuchs M, Iosub S, Bingol N, et al. Palpebral fissure size revisited. F Pediatr 1980;96:77-9.

13 Guimaraes FC, Cruz AV. Palpebral fissure height and downgaze in patients with Graves upper eyelid retraction and congenital blepharoptosis. Ophthalmology 1995;102: 1218-22.

14 Borgen HG, Franti CE, Wilmarth SS. Normal variations of the position of the eye in the orbit. Ophthalmology 1986;93: $1072-7$

15 Mulliken JB, Godwin SL, Pracharktam N. The concept of sagittal orbital-globe relationship in craniofacial surgery. Plast Reconstr Surg 1996;97:700-6.

16 Imai K, Tajima S. Measurement of normal eyeball position and its application for evaluation of exophthalmos in craniofacial synostosis. Plast Reconstr Surg 1993;92:588-92.

17 Yeatts P. Measurements of globe position in complex orbital fractures. II. Patient evaluation using a modified exophthalmometer. Ophthal Plast Reconstr Surg 1992;8:119-25.

18 Camirand A. Canthopexy and transconjunctival blepharoplasty are preferable to lower blepharoplasty. Can f Plast Surg 1994;1:184-7.

19 Camirand A, Doucet J. Surgical advances. A comprehensive approach to surgical juvenation of the eyes. Aest Plast Surg 1996;20:15-22.

20 González-Ulloa M, Stevens E. The senile eyelid. Aesthetic correction. Arch Ophthalmol 1967;78:365-68.

21 Benger RS, Musch DC. A comparative study of eyelid parameters in involutional entropion. Ophthal Plast Reconstr Surg 1989;5:281-7.

22 Collin JRO, Rathbun JE. Involutional entropion. A review with evaluation of a procedure. Arch Ophthalmol 1978;96: 1058-64. 